\title{
Evaluation of energetic particle parameters in the near-Earth magnetotail derived from flux asymmetry observations
}

\author{
Z. Y. Pu ${ }^{1,2}$, R. H. W. Friedel ${ }^{1}$, A. Korth ${ }^{1}$, Q. G. Zong ${ }^{1}$, Z. X. Chen ${ }^{2}$, A. Roux ${ }^{3}$, S. Perraut ${ }^{3}$ \\ ${ }^{1}$ Max-Planck-Institut für Aeronomie, D-37191 Katlenburg-Lindau, Germany \\ 2 Department of Geophysics, University of Peking, Beijing, China \\ ${ }^{3}$ CETP/UVSQ, Vélizy, France
}

Received: 30 April 1997 / Revised: 3 September 1997 / Accepted: 11 September 1997

\begin{abstract}
The flux asymmetries measured by spectrometers on board spacecraft contain information on particle parameters. The net flux intensity (NFI) method provides a tool to evaluate these parameters. The NFI method is valid when both the spin period of the spacecraft and the time resolution of the particle spectrometers are much shorter than the characteristic time-scale of the particle flux variations. We apply the NFI analysis to the flux asymmetry measurements made by GEOS 2 at the nightside geosynchronous orbit in the late substorm growth phase. The cross-tail current of energetic ions, their pressure gradient and average drift velocity, as well as a field-aligned flows are investigated. Current disruption at substorm onset and the "convection surge" mechanism during dipolarization of the magnetic field are directly observed.
\end{abstract}

Key words. Flux asymmetry · Net flux intensity · GEOS 2 - Energetic particles

\section{Introduction}

Quantitative measurements of particle and magnetic field parameters are essentials of magnetospheric research. Recently it became clear that the near-Earth magnetotail plays an important role in the magnetospheric substorm development (Roux et al., 1991; Korth et al., 1991; Atkinson, 1991; Lui, 1991a,b). It has been suggested that during the substorm growth phase the increased tail flaring and inward intrusion of the crosstail current lead to an increase in current density in this region, while the sudden reduction of this current along with current diversion along the field lines into the

Correspondence to: Dr. A. Korth

e-mail: korth@sprotte.mpae.gwdg.de ionosphere causes the onset of the substorm expansion phase (Lopez, 1993; Lui, 1991a,b), followed by energization and injection of energetic ions and electrons (Mauk and Meng, 1987). Quantitative investigations of particle and magnetic field parameters in the near-Earth magnetotail are hence of crucial importance in the investigation of these processes.

A statistical study of GEOS-2 observations (Korth et al., 1991; $\mathrm{Pu}$ et al., 1992) showed that the number density of energetic ions $\left(N_{i,(e n)}\right)$ with energy from $\sim 27$ to $\sim 400 \mathrm{keV}$ at nightside geosynchronous orbit is in the range of $0.2 \mathrm{~cm}^{-3}$ during quiet times, and $\sim 0.3$ to $\sim 1.0 \mathrm{~cm}^{-3}$ during substorm periods. The number density of energetic electrons with energy from $\sim 20$ to $\sim 200 \mathrm{keV}$ is usually more than one order less than the energetic ions. Since plasma densities within the plasma sheet are $\sim 0.1-1 \mathrm{~cm}^{-3}$ (Frank, 1985), the charge neutrality condition then indicates that the number density of thermal electrons of plasmasheet origin $N_{e,(t h)} \sim N_{i,(e n)}$, and $N_{i,(t h)}$ of thermal ions is less than or around $N_{i,(e n)}$. Plasma $\beta$ is attributed mainly to the energetic ions, and is typically $\sim 0.1$ during quiet times and $\geq 1$ during substorm active phases. These statistical results imply that the dynamics of energetic ions may well dominate the physical processes in this region. On the other hand, quantitative features of vector parameters, such as the cross-tail current, field-aligned current, drift velocity $\left(\mathbf{V}_{d}\right)$, and pressure gradient $(\nabla P)$ of energetic particles, are less well understood. In general it is difficult to measure the aforementioned vector quantities with one spacecraft. However, the European geosynchronous satellite GEOS 2 provides an opportunity to make quantitative estimations of these parameters.

The particle spectrometer S321 on board GEOS 2 (Korth et al., 1978) measures electrons in an integral channel with energies above $22 \mathrm{keV}$ and in 16 differential channels between 14 and $213 \mathrm{keV}$. Ions are detected at energies above $27 \mathrm{keV}$ and in ten energy channels between 28 and $402 \mathrm{keV}$. For both ions and electrons a full energy spectrum is obtained every $5.5 \mathrm{~s}$ over a pitch angle range of about $120^{\circ}$, and the integral flux 
intensities are measured simultaneously in all azimuthal directions with a time resolution of $0.172 \mathrm{~s}$. It has been reported that the ion fluxes show strong azimuthal anisotropies during substorm times (Kremser et al., 1982; Roux et al., 1991; Korth et al., 1991; $\mathrm{Pu}$ et al., 1992).

Meanwhile, flux intensities of particles display a noticeable pitch angle asymmetry in some substorm periods (Kremser et al., 1988).

We will see later that these measured flux asymmetries contain information on aforementioned vector quantities. Previously, the azimuthal asymmetries of ion fluxes in the late growth phase were interpreted in terms of the presence of a pressure gradient (Kremser et al., 1982, 1988; Roux et al., 1991; Korth et al., 1991; $\mathrm{Pu}$ et al., 1992). The method to derive this gradient was originally proposed by Walker et al. (1976) and Kremser et al. (1982). They showed that near the midnight sector, ion fluxes with pitch angle around $90^{\circ}$, measured when the detector points to the east (west), are attributed to the ions with guiding centers being earthward and equatorward (tailward and northward or southward) of the satellite. Nevertheless, no effort was made quantitatively to estimate the relevant parameters. The strong pitch angle asymmetry of electrons prior to some expansion onset has been identified as the signature of field-aligned electron beams (Kremser et al., 1988). A few years ago, using a method similar to that of Walker et al. (1976) and Kremser et al. (1982), $\mathrm{Pu}$ et al. (1992) made an estimation of $\beta$ and the scale length of $\nabla P$ of energetic ions in the late growth phase from GEOS-2 observations. In their calculations they took the lowest energy threshold $K_{1}$ of S321 spectrometer to be zero. This yielded an inaccuracy in their evaluation.

In the present paper we extend previous studies using the net flux intensity (NFI) analysis to the GEOS-2 ion flux asymmetry measurements. The lowest energy threshold of $K_{1}=27 \mathrm{keV}$ is adopted. We evaluate the cross-field flow intensity of energetic ions at the GEOS 2 orbit prior to substorm expansion onsets, their crossfield current density and pressure gradient, as well as field-aligned flows. Cross-tail current disruption at substorm onsets and the convection surge mechanism during dipolarization of the magnetic field are directly observed.

\section{Net flux intensity method}

\subsection{NFI analysis of measured particles}

Suppose that the spectrometer measures particles with energy $K \geq K_{1}$ and that the energetic particles that may be interesting to our problems possess a lowest energy threshold of $K_{0}$ with $K_{0}<K_{1}$. Suppose also that the magnetic field $\mathbf{B}$ is perpendicular upwards and that the maximum and minimum flux intensities are observed when the detector is directed subsequently to the $-y$ and $+y$ directions as shown in Fig. 1a.

If both the spin period of the spacecraft and the time resolution of the spectrometers are much shorter than
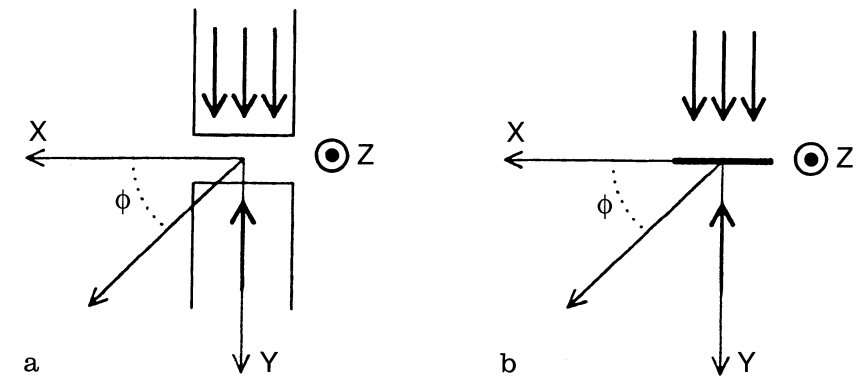

Fig. 1. The NFI principle

the characteristic time-scale of particle flux variations, the maximum azimuthal asymmetry of the integral flux $\Delta J_{y}=J_{+}-J_{-}$approximately denotes the difference of the number of measured particles moving across the unit $(x, z)$ plane per second from the upward side $(\pi<\phi<2 \pi)$ and that from the downward side $(0<\phi<\pi)$, as shown in Fig. 1b, where

$$
\Delta J_{+}=\int_{\pi}^{2 \pi} J(\phi) \cos \phi \mathrm{d} \phi
$$

and

$\Delta J_{-}=\int_{0}^{\pi} J(\phi) \cos \phi \mathrm{d} \phi$.

In this case $\Delta J_{y}$ is basically equivalent to the NFI of measured particles across the $(x, z)$-plane at the same place, i.e., the "effective number" of the particles moving across the unit $(x, z)$-plane from the upward side within unit time. Thus, $\Delta J_{y}$ can be approximately expressed as

$\Delta J_{y} \approx\left\langle N V_{y}\right\rangle=N\left\langle V_{y}\right\rangle$,

where $N$ is the number density of measured particles and $\left\langle V_{y}\right\rangle$ is the averaged "effective" flow velocity of the measured particles. Since $N$ can be derived from the particle spectrometer data on board the spacecraft, we then obtain the effective flow velocity of the particles:

$\left\langle V_{y}\right\rangle \approx \frac{\Delta J_{y}}{N}$.

Furthermore, it is easy to see that

$j_{\perp, s}=q_{s} N_{s}\left\langle V_{y, s}\right\rangle$, where

$q_{s}$ is the charge of an individual particle; $\perp, s$, indicates the cross-field current density contributed by the measured particles (the species of which is denoted by subscript $s$ ). Therefore, by evaluating the maximum NFI with respect to different azimuthal angles, we can find the direction and magnitude of both the effective flow velocity of the particles and the relevant cross-field current density.

The NFI concept can be applied to pitch angle asymmetries as well. Here the symmetry and asymmetry are defined with respect to the pitch angles $\alpha$ and $180^{\circ}-\alpha$. A conventional anisotropic pitch angle distribution may be symmetric relative to the plane perpen- 
dicular to the local magnetic field $\mathbf{B}$. When the total flux in the direction parallel to $\mathbf{B}$ is not identical to that antiparallel to $\mathbf{B}$, a pitch angle asymmetry appears, which leads to a nonzero field-aligned net flux $\Delta J_{\|}$.

So far we have discussed the concept of the NFI in a general way. Naturally, this method is valid only when both the spin period of the spacecraft and the time resolution of the particle spectrometers are much shorter than the characteristic time-scale of the particle flux variations. In this situation the results given by the NFI analysis are approximately identical to those obtained when the detector measures the instantaneous distribution of particles $f(\mathbf{r}, \mathbf{v} ; t)$ (Lundin et al., 1982). While using the NFI method to derive $\Delta J_{y}$ and $\Delta J_{\|}$based on observed asymmetries, there is no need to consider microscopically the behavior and orbits of measured particles.

However, for making quantitative estimations of $\mathbf{j}_{\perp, s},\left\langle V_{y, s}\right\rangle, \nabla P$ contributed by all particles with energy $K \geq K_{0}$, we have to make reasonable simplifications and assumptions.

\subsection{NFI analysis of particles with a shifted Maxwellian distribution}

GEOS-2 observations show that in the near-Earth tail the energy spectra of energetic particles are often approximately composed of a certain number of shifted bi-Maxwellian components, except those in the highenergy tail (Kappa distribution). Figure 2b gives an example which shows that sometimes ions found in most energy channels basically satisfy a Maxwellian-like

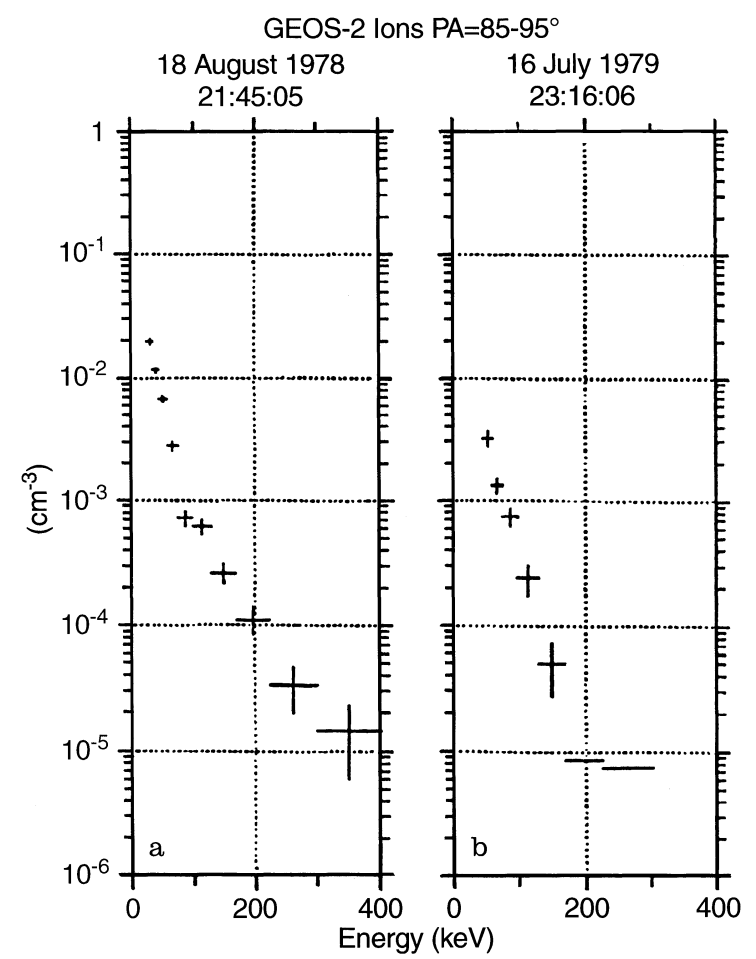

Fig. 2a,b. Examples of $90^{\circ}$ pitch angle ion spectra as measured by S321 on GEOS 2; a for 18 August 1978 and b for 16 July 1979 distribution, while Fig. 2a shows that more generally there are two or more ion populations, each of which possesses a Maxwellian-type distribution.

Note that the pitch angle range of ions plotted in Fig. $2 \mathrm{a}, \mathrm{b}$ is $85^{\circ}-95^{\circ}$, while in general the temperature of particles is anisotropic, i.e., $T_{\perp} \neq T_{\|}$. Note also that the flux intensities shown in Fig. 2a,b are averaged over $2 \pi$ azimuthal angles, whereas in many cases the fluxes exhibit azimuthal asymmetries which are related to the drift velocity of particles. Based on these observations and arguments, we assume that the distribution function of energetic ions in the near-Earth magnetotail may be approximately expressed by a sum of shifted bi-Maxwellian components:

$$
\begin{aligned}
f(x, \mathbf{v})= & \sum_{j} f_{j}(x, \mathbf{v}) \quad(j=0,1,2, \ldots) \\
f_{j}(x, \mathbf{v})= & \frac{N_{j}(x)}{(2 \pi)^{3 / 2} w_{\|, j} w_{\perp, j}^{2}} \exp \left(-\frac{v_{\|}^{2}}{2 w_{\|, j}^{2}}\right) \\
& \times \exp \left(-\frac{v_{x}^{2}+\left(v_{y}-V_{d j}\right)^{2}}{2 w_{\perp, j}^{2}}\right) \\
& \left(K_{j 1}<K<K_{j N}\right)
\end{aligned}
$$

where $j=0,1, \ldots$ indicates the first, second, $\ldots$ shiftedMaxwellian component, $K$ stands for the kinetic energy, $w_{\perp, j}^{2}=T_{\perp, j} / m$ and $w_{\|, j}^{2}=T_{\|, j} / m, w_{\perp, j}$ and $w_{\|, j}$ represent the thermal speed of ions in the direction perpendicular and parallel to the magnetic field, respectively; $V_{d j}$ is the overall or average drift speed of the particles. In writing Eq. (5) we have assumed that ions drift along the $y$-axis. Physically, the vector $\mathbf{V}_{d j}$ should be composed of the $\mathbf{E} \times \mathbf{B}$ drift $\left(\mathbf{V}_{E}\right)$, the diamagnetic drift $\left(\mathbf{V}_{d m}\right)$, and the anisotropy-curvature drift $\left(\mathbf{V}_{a c}\right)$ (Northrop, 1963; Woods, 1987):

$$
\begin{aligned}
\mathbf{V}_{d j}= & \mathbf{V}_{E}+\mathbf{V}_{d m}+\mathbf{V}_{a c} \\
= & \frac{\mathbf{E} \times \mathbf{B}}{B^{2}}+\frac{\mathbf{B} \times \nabla P_{\perp, j}}{N_{j} q_{j} B^{2}} \\
& +\frac{\left(P_{\|, j}-P_{\perp, j}\right)}{N_{j} q_{j} B^{2}} \frac{\mathbf{B} \times(\mathbf{B} \cdot \nabla) \mathbf{B}}{B^{2}}
\end{aligned}
$$

It is noteworthy that during the substorm growth phase, $E_{\perp}$ in the near-Earth magnetotail is in general too weak to produce $V_{E}$ drift comparable to $V_{d m}$ of energetic ions; and often (especially just prior to the expansion phase onset) the pressures of ions and electrons are nearly isotropic (Lui et al., 1987; Pu et al., 1992). In this case,

$$
\begin{aligned}
V_{d j} \approx \frac{\left|\nabla P_{j}\right|}{N_{j} q_{j} B} & \\
f_{j}(x, \mathbf{v})= & \frac{N_{j}(x)}{(2 \pi)^{3 / 2} w_{j}^{3}} \\
& \times \exp \left(-\frac{v_{x}^{2}+\left(v_{y}-V_{d m}\right)^{2}+v_{\|}^{2}}{2 w_{j}^{2}}\right) .
\end{aligned}
$$


In this paper we only discuss the simplest case: the single shifted bi-Maxwellian distribution. It is straightforward to extend the derivation to the multiple Maxwellian population situation. For present purposes we are only interested in energetic ions. Since the typical particle energy of plasmasheet population ranges from 0.5 to $5 \mathrm{keV}$ for ions (Frank, 1985), we set somehow artificially a lowest energy threshold $K_{i, 0} \approx 5 \mathrm{keV}$ for Maxwellian energetic ions. To simplify the analysis, it is assumed that $V_{d} \ll w_{\perp}$. We will see later that this is true for energetic ions at the nightside geosynchronous orbit in the late substorm growth phase. It is easy to see that in this case the number density and thermal pressure of ions with $K>K_{0}$ are, respectively, expressed as

$N_{0}=N\left[1-\operatorname{erf}\left(\frac{v_{0}}{\sqrt{2} w_{\perp}}\right)+\sqrt{\frac{2}{\pi}} \frac{v_{0}}{w_{\perp}} \exp \left(-\frac{v_{0}^{2}}{2 w_{\perp}^{2}}\right)\right]$,

and

$$
\begin{aligned}
P_{0}= & \frac{4 N k T}{3 \sqrt{\pi}}\left[x_{0}^{3} \exp \left(-x_{0}^{2}\right)+\frac{3}{2} x_{0} \exp \left(-x_{0}^{2}\right)\right. \\
& \left.+\frac{3 \sqrt{\pi}}{4}\left(1-\operatorname{erf}\left(x_{0}\right)\right)\right],
\end{aligned}
$$

where the geometry of Fig. $1 \mathrm{~b}$ was used, $v_{0}=\sqrt{2 K_{0} / m}$, and

$\operatorname{erf}(\alpha)=\frac{2}{\sqrt{\pi}} \int_{0}^{\alpha} \exp \left(-x^{2}\right) \mathrm{d} x$

is the error function.

Furthermore, the maximum net cross-field flux intensity of the Maxwellian ions is written as

$\Delta F_{y}=F_{\max }-F_{\min }$,

where

$$
\begin{aligned}
F_{\max } \approx & \int_{-\infty}^{\infty} \int_{v_{0}}^{\infty} \int_{-\infty}^{\infty} v_{y} f\left(x, v_{x}, v_{y}, v_{\|}\right) \mathrm{d} v_{x} \mathrm{~d} v_{y} \mathrm{~d} v_{\|} \\
= & \frac{N}{(2 \pi)^{1 / 2} w_{\perp}} \int_{v_{0}}^{\infty} \exp \left(-\frac{\left(v-V_{d}\right)^{2}}{2 w_{\perp}^{2}}\right) \mathrm{d} v_{y} \\
= & \frac{N w_{\perp}}{\sqrt{2 \pi}} \exp \left(-\frac{\left(v_{0}-V_{d}\right)^{2}}{2 w_{\perp}^{2}}\right) \\
& +\frac{N V_{d}}{2}\left[1-\operatorname{erf}\left(\frac{v_{0}-V_{d}}{\sqrt{2} w_{\perp}}\right)\right]
\end{aligned}
$$

and

$$
\begin{aligned}
F_{\min } & \approx \int_{-\infty}^{\infty} \int_{-v_{0}}^{-\infty} \int_{-\infty}^{\infty} v_{y} f\left(x, v_{x}, v_{y}, v_{\|}\right) \mathrm{d} v_{x} \mathrm{~d} v_{y} \mathrm{~d} v_{\|} \\
& =\frac{N}{(2 \pi)^{1 / 2} w_{\perp}} \int_{-v_{0}}^{-\infty} \exp \left(-\frac{\left(v-V_{d}\right)^{2}}{2 w_{\perp}^{2}}\right) \mathrm{d} v_{y}
\end{aligned}
$$

$$
\begin{aligned}
= & \frac{N w_{\perp}}{\sqrt{2 \pi}} \exp \left(-\frac{\left(v_{0}+V_{d}\right)^{2}}{2 w_{\perp}^{2}}\right) \\
& -\frac{N V_{d}}{2}\left[1-\operatorname{erf}\left(\frac{v_{0}+V_{d}}{\sqrt{2} w_{\perp}}\right)\right] .
\end{aligned}
$$

It can be seen that as $v_{0} \rightarrow 0, N_{0} \rightarrow N, P_{0} \rightarrow N k T$, and $\Delta F_{y} \rightarrow N V_{d}$. In general, $N_{0}<N, P_{0}<N k T$, and $\Delta F_{y}<N V_{d}$.

To calculate $N_{0}, P_{0}$, and $\Delta F_{y}$, it is necessary first to derive $N, w_{\perp}$, and $V_{d}$. This can be done as follows. For a detector with lower energy threshold, $K_{1}>K_{0}$, one can derive from measurements the number density $N_{1}$, thermal pressure $P_{1}$ of particles with energy $K \geq K_{1}$. On the other hand, it can readily be obtained that

$$
\begin{aligned}
N_{1}= & N\left[1-\operatorname{erf}\left(x_{1}\right)+\frac{2}{\sqrt{\pi}} x_{1} \exp \left(-x_{1}^{2}\right)\right], \\
P_{1}= & \frac{4 N k T}{3 \sqrt{\pi}}\left[x_{1}^{3} \exp \left(-x_{1}^{2}\right)+\frac{3}{2} x_{1} \exp \left(-x_{1}^{2}\right)\right. \\
& \left.+\frac{3 \sqrt{\pi}}{4}\left(1-\operatorname{erf}\left(x_{1}\right)\right)\right],
\end{aligned}
$$

where $x_{1}=\frac{v_{1}}{\sqrt{\pi} w_{\perp}}, v_{1}=\sqrt{2 K_{1} / m}$. Thus $N$ and $w_{\perp}$ can be found by numerically solving Eqs. (14) and (15). Furthermore, $V_{d}$ can be estimated from the azimuthal anisotropy of integral fluxes $J_{l}\left(\alpha \approx 90^{\circ}, \phi_{i}\right)$, where $l=1,2, \ldots, 16 ; \phi_{1} \approx 10^{\circ}, \phi_{2} \approx 30^{\circ}$, etc. It is easy to see that

$$
\begin{aligned}
J_{\max } \approx & \int_{v_{1}}^{\infty} v f\left(v^{2}, \alpha \approx 90^{\circ}, \phi \approx 90^{\circ}\right) v^{2} \mathrm{~d} v \\
= & \frac{N}{(2 \pi)^{3 / 2} w_{\|} w_{\perp}^{2}} \int_{v_{1}}^{\infty} v^{3} \exp \left[-\frac{\left(v-V_{d}\right)^{2}}{2 w_{\perp}^{2}}\right] \mathrm{d} v \\
= & \frac{\eta N w_{\perp}}{\sqrt{2} \pi}\left\{\left[\left(\frac{V_{d}}{\sqrt{2} w_{\perp}}\right)^{3}+\frac{3}{2} \frac{V_{d}}{\sqrt{2} w_{\perp}}\right]\right. \\
& \times\left[1-\operatorname{erf}\left(\frac{v_{1}-V_{d}}{\sqrt{2} w_{\perp}}\right)\right] \\
& +\left[\frac{3}{\sqrt{\pi}}\left(\frac{V_{d}}{\sqrt{2} w_{\perp}}\right)^{2}+\frac{3}{\sqrt{\pi}} \frac{V_{d}}{\sqrt{2} w_{\perp}}\left(\frac{v_{1}-V_{d}}{\sqrt{2} w_{\perp}}\right)\right. \\
& \left.+\frac{1}{\sqrt{\pi}}+\frac{1}{\sqrt{\pi}}\left(\frac{v_{1}-V_{d}}{\sqrt{2} w_{\perp}}\right)^{2}\right] \\
& \left.\times \exp \left(-\frac{\left(v_{1}-V_{d}\right)^{2}}{2 w_{\perp}^{2}}\right)\right\}
\end{aligned}
$$

and

$$
\begin{aligned}
J_{\min } & \approx \int_{v_{1}}^{\infty} v f\left(v^{2}, \alpha \approx 90^{\circ}, \phi \approx 270^{\circ}\right) v^{2} \mathrm{~d} v \\
& =\frac{N}{(2 \pi)^{3 / 2} w_{\|} w_{\perp}^{2}} \int_{v_{1}}^{\infty} v^{3} \exp \left(-\frac{\left(v+V_{d}\right)^{2}}{2 w_{\perp}^{2}}\right) \mathrm{d} v
\end{aligned}
$$




$$
\begin{aligned}
= & -\frac{\eta N w_{\perp}}{\sqrt{2} \pi}\left\{\left[\left(\frac{V_{d}}{\sqrt{2} w_{\perp}}\right)^{3}+\frac{3}{2} \frac{V_{d}}{\sqrt{2} w_{\perp}}\right]\right. \\
& \times\left[1-\operatorname{erf}\left(\frac{v_{1}+V_{d}}{\sqrt{2} w_{\perp}}\right)\right] \\
& +\left[\frac{3}{\sqrt{\pi}}\left(\frac{V_{d}}{\sqrt{2} w_{\perp}}\right)^{2}-\frac{3}{\sqrt{\pi}} \frac{V_{d}}{\sqrt{2} w_{\perp}}\left(\frac{v_{1}+V_{d}}{\sqrt{2} w_{\perp}}\right)\right. \\
& \left.+\frac{1}{\sqrt{\pi}}+\frac{1}{\sqrt{\pi}}\left(\frac{v_{1}+V_{d}}{\sqrt{2} w_{\perp}}\right)^{2}\right] \\
& \left.\times \exp \left(-\frac{\left(v_{1}+V_{d}\right)^{2}}{2 w_{\perp}^{2}}\right)\right\} .
\end{aligned}
$$

It can be demonstrated that when $V_{d}>0, \Delta F_{y}>$ $\Delta J_{y}>0$.

Now define the ratio of

$M=\frac{\Delta J_{y}}{\left\langle J_{y}\right\rangle}=\frac{J_{\max }-J_{\min }}{\left(J_{\max }+J_{\min }\right) / 2}$,

which can be obtained from the observed flux asymmetry data; then the drift velocity $V_{d}$ can be evaluated by numerically solving Eq. (18) together with Eqs. (16) and (17). The cross-field current contributed by energetic ions with $K \geq K_{0}$ can then be quantitatively estimated if no strong electric fields exist. In this situation, if particle pressure is isotropic, one has

$\frac{V_{d}}{w_{\perp}} \approx \frac{V_{d m}}{w_{\perp}} \approx \frac{r_{g}}{L_{P}}$,

where $r_{g}=w_{\perp} / \Omega$ stands for the average gyroradius of particles, $\Omega=q B / m$ denotes the particle gyrofrequency, and $L_{P}$ is the scale length of the pressure gradient.

It is important to note that when $v_{1} \sim w_{\perp}, M$ may be considerably greater than $V_{d} / w_{\perp}$. If one uses $M \approx V_{d} / w_{\perp}$ (which holds if $v_{1}=0$ ) to evaluate $V_{d}$ and $L_{p}$, this will yield a significant inaccuracy in the estimation.

It should be mentioned that by using the collisionless two-fluid approach, Mitchell et al. (1986) developed two techniques (the Compton-Getting transform method and the distribution function shift method) to analyze bulk flows, $\mathbf{E} \times \mathbf{B}$ convection, and pressure gradients of energetic ions from the anisotropy of ion distributions measured by the MEPI experiment on the ISEE-1 spacecraft. It was mentioned that one could distinguish between spatial gradient and the convection drift in the energetic ion motion by using their method. We will see later that the NFI method developed in this paper is suitable and much easier to use for evaluating parameters of shifted Maxwellian ions in the near-Earth magnetotail when the $\mathbf{E} \times \mathbf{B}$ convection is much less than the gradient drift motion.

To end this subsection, it should be pointed out that the assumption of Eq. (5) and Eq. (6) are based on the particle (or two-fluid) dynamics. On the other hand, the NFI analysis may also be used on the basis of the guidingcenter approximation. This is done when one replaces $x$, the particle position, in Eq. (5) with $X$, the position of the guiding center, where $X \approx x+v_{y} / \Omega$. Nevertheless, it can be proved that, while transforming from the guidingcenter formalism to the particle formalism, to the first order, one eventually gets Eqs. (5) and (6), and thus the same results as we obtain in this work. This we will show in detail in an another paper.

\section{Study of substorm expansion onsets with the NFI analysis}

\subsection{Disruption of the cross-tail current}

We selected two substorms of 26 February 1979 and 25 January 1979 observed by GEOS 2 as case-studies. Figures 3 and 4 show, respectively, time variations of the magnetic field and integral flux intensities of ions with pitch angles around $90^{\circ}$ measured when detectors of spectrometer S321 pointed to west-east and earthward-tailward for these two substorms.

The expansion phase onsets indicated by the onset of Pi2 pulsation as measured by mid-latitude stations are marked by vertical dashed lines in Figs. 3 and 4. Three striking features are immediately seen:

1. Distinct azimuthal asymmetries $\left(\Delta J_{y, i}\right)$ appear in the east-west flux intensities of energetic ions before onsets.

2. 1-3 min prior to the onset $\Delta J_{y, i}$ reduces and tends to zero.

3. Right after the onset, during the expansion and recovery phases of the first two events, both west-east and earthward-tailward components of $\Delta J_{i}$ become highly turbulent.

It is worthwhile recalling that these three features were found in our previous statistical study of 22 substorm events, and no visible asymmetries occur on magnetically quiet days (Korth et al., 1991; Pu et al., 1992). The existence of prominent $\Delta J_{y, i}>0$ in the late growth phase indicates the occurrence of enhanced cross-tail ion currents in the near-Earth magnetotail at these times, and the reduction and temporary disappearance of $\Delta J_{y, i}$ just prior and at the onset mean that the cross-tail current of energetic ions undergoes disruption. Note that during the growth phase at the nightside synchronous orbit the major current carriers are energetic ions (Lui et al., 1987; Pu et al., 1992) and that the $V_{E}$ drift is considerably smaller than $V_{d}$.

Therefore, the fact that $\Delta J_{y}$ rapidly decreases and temporarily disappears just manifests the collapse of the near-Earth current sheet while the dipolarization of the magnetic field occurs.

\subsection{Evaluation of energetic ion parameters at the expansion onsets}

Now we make quantitative estimations of energetic ion parameters for these two events under the assumption of the single shifted bi-Maxwellian distribution. The energy threshold of the GEOS-2 ion spectrometer for the integral flux $K_{1}=27 \mathrm{keV}$. One to three minutes prior to 


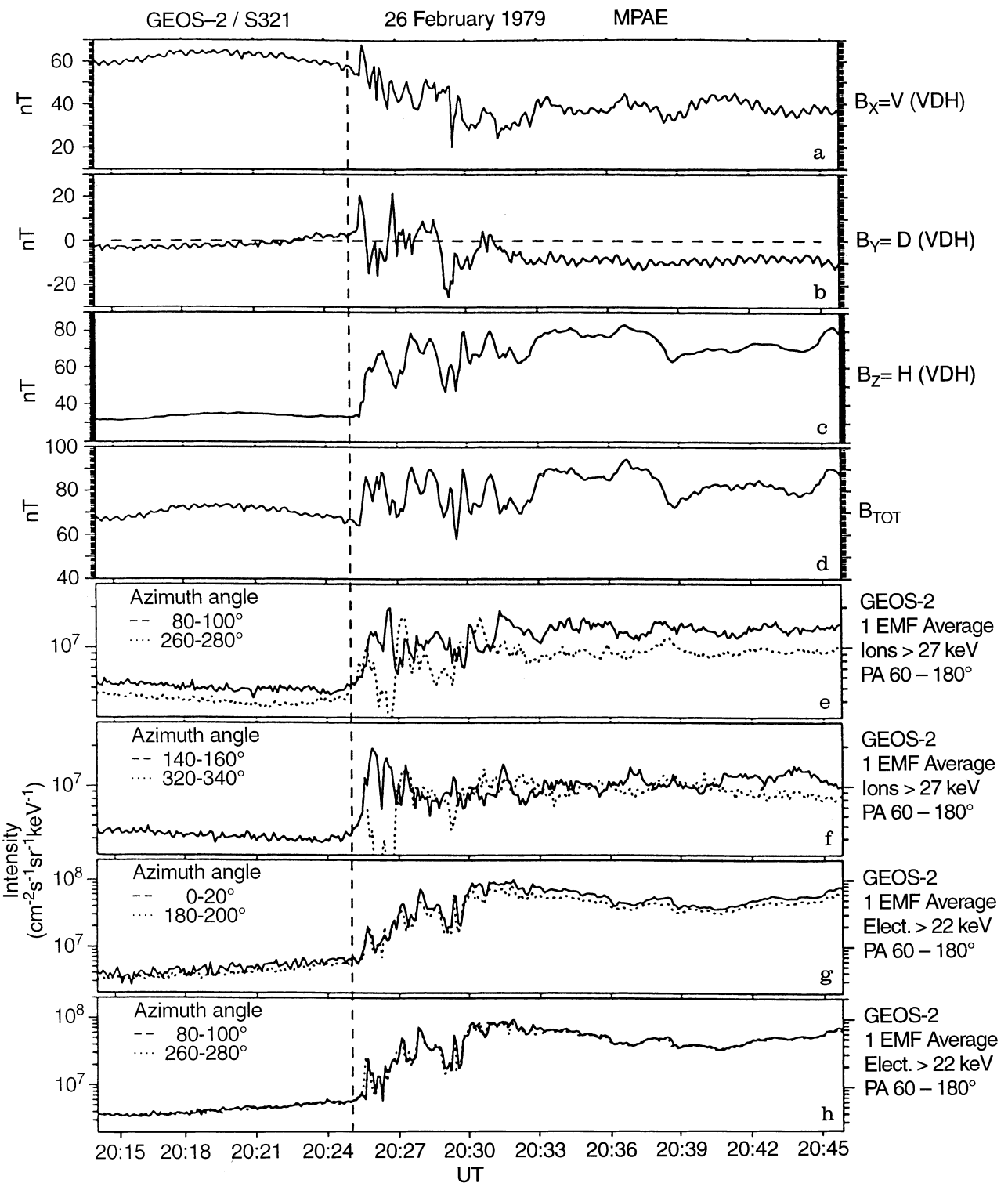

Fig. 3a-h. Event on 26 February 1979: a-d magnetic field in VDH components, $\mathbf{e}$ and $\mathbf{f}$ integral ions at two opposite azimuths, $\mathbf{g}$ and $\mathbf{h}$ integral electrons at the opposite azimuths the expansion phase onset of these two substorms, $E_{i}$, the energy density of energetic ions, and the maximum value of $M_{i}$ are $13.6 \mathrm{keV} \mathrm{cm}^{-3}$ and 0.35 , and $9.9 \mathrm{keV} \mathrm{cm}^{-3}$ and 0.33 , respectively. The anisotropy parameter $A_{i}=P_{\|, i} / P_{\perp, i} \approx 1$.

Numerical results are listed in Table 1 , where $N_{1, i}$ is ion number density observed by S321 with $K>27 \mathrm{keV}, N_{0, i}$ denotes the number density of ions with $K>5 \mathrm{keV}$, and $L_{P, i}^{0}$ refers to the scale length of the pressure gradient of energetic ions at the magnetic equator. In calculating $L_{P, i}^{0}$ the ratio of $B_{0} / B \approx 0.6$ has been used, where $B_{0}$ stands for the magnitude of $B$ at the equator along the same field line (Pu et al., 1992). Note that the cross-tail current densities of energetic ions in the late growth phase of these two substorms are comparable to the current densities of energetic ions in the nightside equatorial region at $6<L<8$ found by AMPTE/CCE during two geomagnetic storms in September 1984 (Lui et al., 1987). Note also that the scale length of the pressure gradient of energetic ions built up in the late growth phase of these two substorms is $\sim 1 \mathrm{R}_{\mathrm{E}}$, which is consistent with the fact that the radial scale length of the substorm onset area near the geosynchronous region was found to be of the order of $1 \mathrm{R}_{\mathrm{E}}$ or less (Ohtani et al., 1991). Note furthermore that during the growth phase, $E_{\perp}$ in the near-Earth magnetotail is in general too weak to produce a $V_{E}$ drift which can be comparable with $V_{d m, i}$. For instance, the typical magnitude of the magnetic field in the growth phase is

Table 1. Energetic ion parameters at the GEOS-2 position prior to substorm onset

\begin{tabular}{lllllllll}
\hline Date (1979) & $E_{i} \mathrm{keV} \mathrm{cm}^{-3}$ & $N_{1, i} \mathrm{~cm}^{-3}$ & $N_{0, i} \mathrm{~cm}^{-3}$ & $M_{i}$ & $V_{d, i} / w_{i}$ & $V_{d, i} \mathrm{~km} \mathrm{~s}^{-1}$ & $L_{P, i}^{0} \mathrm{~km}_{\perp, i} \mathrm{Cs}^{-1} \mathrm{~m}^{-2}$ \\
\hline 26 February & 9.9 & 0.27 & 0.83 & 0.33 & $4.3 \times 10^{-2}$ & 60.3 & 5710 & $0.57 \times 10^{-8}$ \\
25 January & 13.6 & 0.30 & 0.56 & 0.35 & $5.5 \times 10^{-2}$ & 98.5 & 5544 & $0.86 \times 10^{-8}$ \\
\hline
\end{tabular}




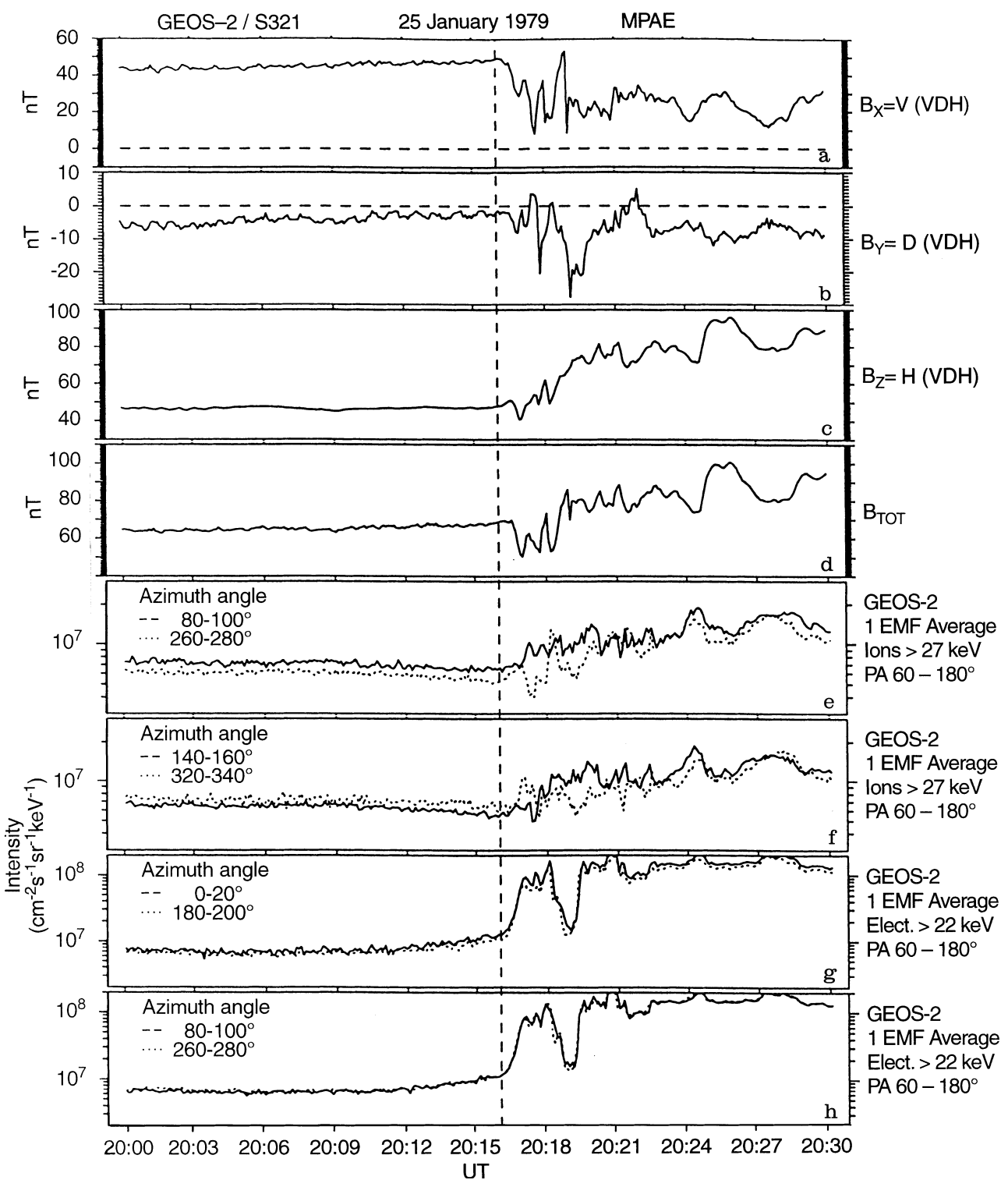

Fig. 4a-h. Event on 25 January 1979: a-d magnetic field in VDH components, $\mathbf{e}$ and $\mathbf{f}$ integral ions at two opposite azimuths, $\mathbf{g}$ and $\mathbf{h}$ integral electrons at the opposite azimuths

$B \approx 70 \mathrm{nT}$. A $V_{E}$ drift of $75 \mathrm{~km} \mathrm{~s}^{-1}$ requires an electric field $E_{\perp}$ which is $\approx 5.5 \mathrm{mV} \mathrm{m}^{-1}$. This can only be the case when strong inductive electric fields appear. Finally, it is worthwhile noticing from Table 1 that $V_{d, i} \ll w_{i}$, in agreement with the assumption made at the beginning of the last subsection.

\subsection{Pitch angle anisotropy during substorm dipolarization}

As can be seen from Fig. 5, the total flux intensity parallel to the magnetic field can be expressed as

$$
\begin{aligned}
J_{\|,+} & =\int_{0}^{\pi / 2} \int_{0}^{2 \pi} \int_{v_{1}}^{\infty} v_{\|} f(\mathbf{v}) \mathrm{d} \mathbf{v} \\
& =\int_{0}^{\pi / 2} F(\alpha) \cos \alpha \sin \alpha \mathrm{d} \alpha,
\end{aligned}
$$

where

$J(\alpha)=\int_{0}^{2 \pi} \int_{K_{1}}^{\infty} f(K, \alpha, \phi) \sqrt{2 K / m} \mathrm{~d} \phi \mathrm{d} K$.

Similarly, the total flux intensity antiparallel to the field lines can be expressed as

$J_{\|,-}=\int_{\pi / 2}^{\pi} F(\alpha) \cos \alpha \sin \alpha \mathrm{d} \alpha$.

Hence the total net field-aligned flux intensity

$$
\begin{aligned}
\Delta, J_{\|} & =J_{\|,+}-J_{\|,-} \\
& =\int_{0}^{\pi / 2}[J(\alpha)-J(\pi-\alpha)] \cos \alpha \sin \alpha \mathrm{d} \alpha,
\end{aligned}
$$




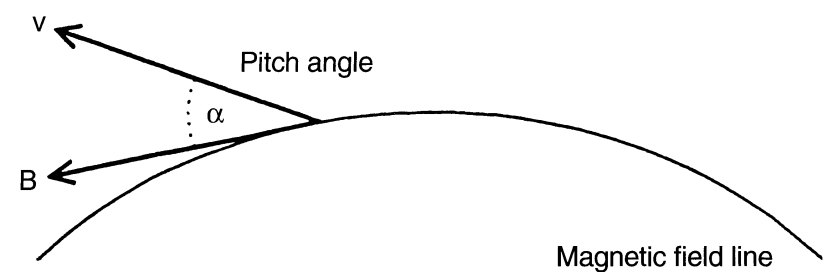

Fig. 5. Definition of pitch angle $\alpha$

or, in terms of fluxes measured in different energy channels

$\Delta J_{\|}=\sum_{i}\left[J\left(\alpha_{i}\right)-J\left(\pi-\alpha_{i}\right)\right] \cos \alpha_{i} \sin \alpha_{i} \Delta \alpha_{i}$

with

$J\left(\alpha_{i}\right)=2 \pi \sum_{l} \sum_{k} J\left(K_{k}, \alpha_{i}, \phi_{l}\right) \Delta K_{k} \Delta \phi_{l}$.
Equations (20) and (21) illustrate how pitch angle asymmetries result in a net field-aligned flux, and hence field-aligned flow. To evaluate the field-aligned flow, measurements covering the full pitch-angle range are needed. Nevertheless, if the whole range is not completely covered, for instance, data are available only for $\alpha_{k}<\alpha<\pi$, the part of the net flux intensity $\int_{\alpha k}^{\pi-\alpha k}(J(\alpha)-J(\pi-\alpha)) \cos \alpha \sin \alpha \mathrm{d} \alpha$, or a comparison of pitch angle asymmetries $J\left(\alpha_{i}\right)-J\left(\pi-\alpha_{i}\right)\left(\alpha_{i}>\alpha_{k}, i=\right.$ $1,2,3, \ldots)$ can give information on the direction as well as on the order of magnitude of the field-aligned flow. Parts a-d of Fig. 6 display pitch angle asymmetries of the integral ion flux intensities in four pitch angle ranges during dipolarization of substorm of 26 February 1979. Electron flux intensities in the pitch angle ranges of $45^{\circ}-55^{\circ}$ and $125^{\circ}-135^{\circ}$ are shown in Fig. 6e for comparison.

The dipolarization time of the substorm of 26 February 1979 is about 1 min, which is much longer than both the spin period of GEOS 2 and the time

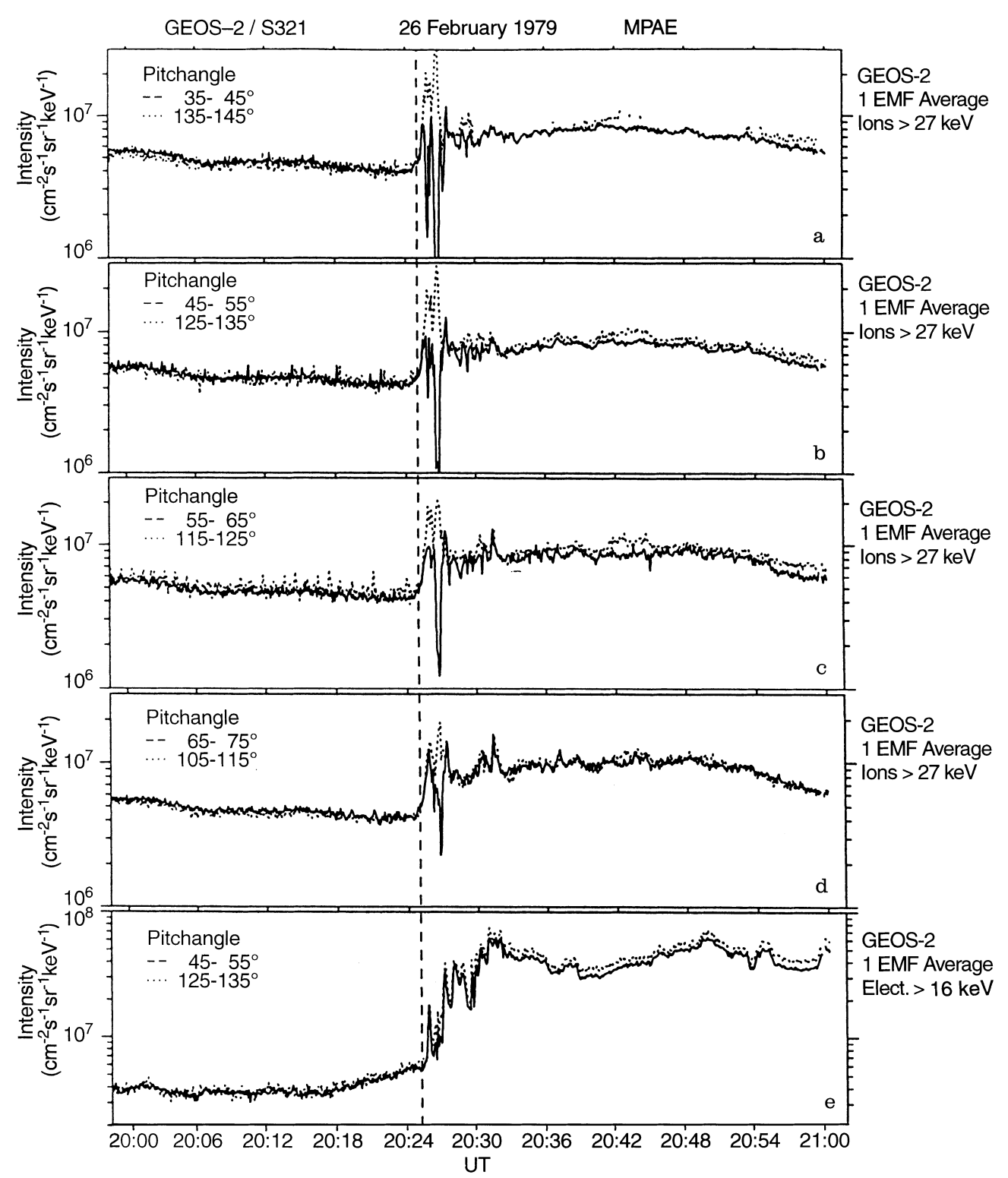

Fig. 6a-e. Ion pitch angle asymmetries for four pitch angle ranges in a-d, and in e for one electron pitch angle range for comparison 
resolution of S321. Therefore, Fig. 6 does make sense and contains meaningful implications. We see that prominent pulses of $\Delta J(\alpha)$ appear right at and right after the expansion phase onset, the magnitude of which increases with decreasing $\alpha$. These pulses obviously manifest the appearance of field-aligned flows. Of particular interest is the fact that these pulses occur simultaneously with the dramatic enhancement of $\Delta J_{\perp, i}$ in the westward directions, while the flux intensities of electrons basically remained symmetric, see Fig. 6e. This indicates that parallel electric fields are not important in the region near the spacecraft, while the inductive westward electric field in connection with the dipolarization of the magnetic field dramatically accelerates ions westward, at the same time causing an earthward $\mathbf{E} \times \mathbf{B}$ convection.

This phenomenon has been known as the "convection surge" mechanism (Quinn and Southwood, 1982; Mauk, 1986; Delcourt et al., 1990) that produces parallel energization of ions during dipolarization of magnetic field at the substorm onset in the current disruption region.

\section{Conclusion}

We have shown that the NFI method provides a useful tool to evaluate a few vector parameters of energetic ions in the near-Earth magnetotail. The NFI method is valid when both the spin period of the spacecraft and the time resolution of the particle spectrometers are much shorter than the characteristic time-scale of the particle flux variations. We apply the NFI analysis to the flux asymmetry observations made by GEOS 2 at the nightside geosynchronous orbit in the late substorm growth phase. The cross-tail current of energetic ions, their pressure gradient and average drift velocity, as well as field-aligned flows, are investigated. Current disruption at substorm onset and the "convection surge" mechanism during dipolarization of the magnetic field are directly observed.

Acknowledgements. We are very grateful to A. Pedersen, Ø. Holter, C. Altman, and T. Bäsinger for helpful discussions. This work has been made possible by the visiting fellowship scheme of the Max Planck Society. The first author is also grateful to the CNSF for their support. Many thanks to F. Both for providing the basic GEOS software support. The particle spectrometer was designed and constructed with financial support from the Max-PlanckGesellschaft zur Förderung der Wissenschaften and the Bundesministerium für Forschung and Technologie through the DFVLR-PT under contract RV14-B12/73 (WRK 243)-SF 21.

Topical Editor K.-H. Glaßmeier thanks two referees for their help in evaluating this paper.

\section{References}

Atkinson, G., A magnetosphere wags the tail model of substorms, in Magnetospheric substorms, Ed. J. R. Kan et al., Geophys Monogr 64, AGU, Washington DC, p. 191, 1991.

Delcourt, D. C., J. A. Sauvaud, and A. Pedersen, Dynamics of single-particle orbits during substorm expansion phase, J. Geophys. Res., 95, 20853, 1990.

Frank, L., Plasmas in the Earth's magnetotail, Space Sci. Rev., 42, 211, 1985.

Korth, A., G. Kremser, and B. Wilken, Observations of substormassociated particle flux variations at $6<l<8$ with GEOS-1, Space Sci. Rev., 22, 501, 1978.

Korth, A., Z. Y. Pu, G. Kremser, and A. Roux, A statistical study of substorm onset conditions at geostationary orbit, in Magnetospheric substorms, Ed. J. R. Kan et al., Geophys. Monogr. 64, AGU, Washington DC, p. 343, 1991.

Kremser, G., et al., Coordinated balloon-satellite observations of energetic particles at the onset of a magnetospheric substorm, J. Geophys. Res., 87, 4445, 1982.

Kremser, G., A. Korth, S. L. Ullaland, S. Perraut, A. Roux, A. Pedersen, R. Schmidt, and P. Tanskanen, Field-aligned beams of energetic electrons $(16 \mathrm{keV}<E<80 \mathrm{keV})$ observed at geosynchronous orbit at substorm onsets, J. Geophys. Res., 93, 14453, 1988.

Lopez, R. E., On the location of the substorm initiation region, Adv. Space. Res., (4) 13, 189, 1993.

Lui, A. T. Y., Extended considerations of a synthesis model for magnetospheric substorms, in Magnetospheric substorms, Ed. J. R. Kan et al., Geophys. Monogr. 64, AGU, Washington DC, p. 43, 1991a.

Lui, A. T. Y., A synthesis of magnetospheric substorm models, J. Geophys. Res., 96, 1849, $1991 \mathrm{~b}$.

Lui, A. T. Y., R. W. McEntire, and S. M. Krimigis, Evolution of the ring current during two geomagnetic storms, J. Geophys. Res., 92, 7459, 1987.

Lundin, R., B. Hultqvist, N. Pissarenko, and A. Zackarov, The plasma mantle, Space Sci. Rev., 31, 247, 1982.

Mauk, B. H., Quantitative modeling of the "convection surge" mechanism of ion acceleration, J. Geophys. Res., 91, 13423, 1986.

Mauk, B. H., and C. I. Meng, Plasma injection during substorms, Phys. Scr., T18, 128, 1987.

Mitchell, D. G., R. Lundin, and D. J. Williams, Analyses of convective flows and spatial gradients in energetic ion observations, J. Geophys. Res., 91, 8827, 1986.

Northrop, T. G., The adiabatic motion of charged particles, WileyInterscience, New York, 1963.

Ohtani, S., K. Takahashi, L. Zanetti, T. A. Potemra, R. W. McEntire, and T. Ijima, Tail current disruption in the geosynchronous region, in Magnetospheric substorms, Ed. J. R. Kan et al., Geophys. Monogr, 64, AGU, Washington DC, p. 191, 1991.

Pu, Z. Y., A. Korth, and G. Kremser, Plasma and magnetic field parameters at substorm onsets derived from GEOS 2 observations, J. Geophys. Res., 97, 19341-19349, 1992.

Quinn, J. M., and D. J. Southwood, Observations of parallel ion energization in the equatorial region, J. Geophys. Res., 87, 10536, 1982.

Roux, A., et al., Plasma sheet instability related to the westward traveling surge, J. Geophys. Res., 96, 19697, 1991.

Walker, R. J., K. N. Erickson, R. J. Swanson, and J. R. Winckler, Substorm-associated particle boundary motion at synchronous orbit, J. Geophys. Res., 81, 5541, 1976.

Woods, L. C., Principles of magnetospheric dynamics, Oxford Science, Oxford, 1987. 